
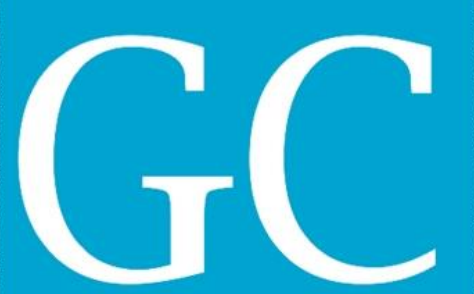

ISSN 2318-8472

v. 07, n. 53,2019

Revista Nacional de

Gerenciamento de Cidades

\title{
Análise bibliométrica de produções científicas de City Information Modelling
}

Bibliometric analysis of scientific productions of City Information Modelling

Análisis bibliométrico de producciones científicas de City Information Modelling

\author{
Letícia de Souza \\ Mestranda, UFSCar, Brasil. \\ Leticia.souza1707@gmail.com \\ Cristiane Bueno \\ Professora Doutora, UFSCar, Brasil. \\ cbueno@ufscar.br
}




\section{RESUMO}

Amorim (2016b) apresenta o City Information Modelling (CIM) como um sistema focado na gestão, no projeto de construções, no planejamento e no monitoramento da cidade, sendo abordado como suporte ao gerenciamento da infraestrutura de uma cidade inteligente. E Xu et al. (2014) define como um sistema para gerenciamento eficiente de cidades com o foco de alcançar compartilhamento de informações sobre vários sistemas da cidade em tempo real, de forma centralizada e acessível afim de melhorar a eficiência geral da gestão urbana. 0 termo foi cunhado inicialmente por Khemlani (2016) vislumbrando uma representação digital da cidade que apoiasse de maneira eficaz as decisões e análises em casos de ocorrência de desastres naturais. Por se tratar de um assunto relativamente novo e não estar plenamente definido, diante disto a pesquisa faz o uso de análise bibliográfica para destacar o progresso das produções cientificas ao longo do tempo, as colaborações entre países, as palavras-chave de maior proeminência e possíveis lacunas de pesquisas que poderiam beneficiar esse estabelecimento. Para tanto, realizouse uma análise bibliométrica sobre o tema CIM. Os dados para o estudo bibliométrico foram coletados nas bases Web of Science e Scopus e foram processados por meio do software VOSviewer. Os resultados demonstraram um crescente número de publicações neste campo, e correlações entre eventos ocorridos a nível mundial, como a reunião da ONU que debatia as Perspectivas da Urbanização Mundial e a Agenda do Desenvolvimento Sustentável de 2030.

PALAVRAS-CHAVE: City Information Modelling, Ferramentas de Gestão Urbana, Cidades Inteligentes.

\section{ABSTRACT}

Amorim (2016b) introduce City Information Modeling (CIM) as a system focused on city management, building design, planning and monitoring, and is approached as support for managing a smart city infrastructure. And Xu et al. (2014) defines it as a system for efficient city management with the focus of achieving real-time, centralized and accessible sharing of information about various city systems in order to improve the overall efficiency of urban management. The term was first coined by Khemlani (2009) envisioning a digital representation of the city that would effectively support decisions and analysis in cases of natural disasters. Because it is a relatively new subject and not fully defined, the research makes use of bibliographic analysis to highlight the progress of scientific productions over time, collaborations between countries, the most prominent keywords and possible research gaps that could benefit this establishment. To this end, a bibliometric analysis on the CIM theme was performed. Data for the bibliometric study were collected from the Web of Science and Scopus databases and were processed using VOSviewer software. The results demonstrated a growing number of publications in this field, and correlations between events occurring worldwide, such as the UN meeting debating the World Urbanization Perspectives and the 2030 Sustainable Development Agenda.

KEYWORDS: City Information Modelling, Urban Management Tools, Smart Cities.

\section{RESUMEN}

Amorim (2016b) presenta City Information Modeling (CIM) como un sistema centrado en la gestión de la ciudad, el diseño de edificios, la planificación y el monitoreo, y se aborda como un soporte para la gestión de una infraestructura de ciudad inteligente. Y Xu y col. (2014) lo define como un sistema para la gestión eficiente de la ciudad con el objetivo de lograr el intercambio de información en tiempo real, centralizado y accesible sobre varios sistemas de la ciudad para mejorar la eficiencia general de la gestión urbana. El término fue acuñado por primera vez por Khemlani (2009) asumiendo una representación digital de la ciudad que apoyaría efectivamente las decisiones y el análisis en caso de desastres naturales. Debido a que es un tema relativamente nuevo y no está completamente definido, la investigación utiliza el análisis bibliográfico para resaltar el progreso de las producciones científicas a lo largo del tiempo, las colaboraciones entre países, las palabras clave más destacadas y posibles vacíos de investigación que podrían beneficiar a este establecimiento. Para ello, se realizó un análisis bibliométrico sobre el tema CIM. Los datos para el estudio bibliométrico se obtuvieron de las bases de datos de Web of Science y Scopus y se procesaron con el software VOSviewer. Los resultados demostraron un número creciente de publicaciones en este campo, y las correlaciones entre los eventos que ocurren en todo el mundo, como la reunión de la ONU que discute las Perspectivas de Urbanización Mundial y la Agenda de Desarrollo Sostenible 2030.

PALABRAS CLAVE: City Information Modelling, Herramientas de Gestión Urbana, Ciudades Inteligentes. 


\section{Revista Nacional de}

Gerenciamento de Cidades

\section{INTRODUÇÃo}

O City Information Modelling (CIM) resultou de uma vontade de proporcionar ferramentas para uma simulação urbana que centralize em tempo real informações e mudanças da cidade. É considerada como de grande importância a funcionalidade de fornecer uma abordagem pluralista de diversos cenários de projetos futuros para que as cidades se desenvolvam de forma integrada e possibilite se tornar verdadeiramente inteligente (BATTY ET AL., 2012).

Sendo esta, uma ferramenta necessária para se compreender a complexidade das cidades devido ao crescimento desenfreado alertada pela Organização das Nações Unidas (ONU) que em torno de 5,1 bilhões de pessoas estará vivendo em cidades até 2050 (ONU, 2012).

Este fenômeno exige atenção às demandas dessa superpopulação em áreas urbanas. Os reflexos desta urbanização abordada por Weiss et al. (2017) são dificuldades na gestão de resíduos sólidos, gestão ineficiente de recursos, contaminação do ar, precarização do sistema de saúde, saturação nas redes de mobilidade, insegurança pública, entre outras dificuldades que restringem a qualidade de vida da população.

Em consequência destas dificuldades, a ONU estabeleceu, na Agenda do Desenvolvimento Sustentável de 2030, alguns objetivos para concretização do conceito de cidades inclusivas, assegurando o direito humano a todos. Para tanto, será necessário grandes intervenções no contexto de cidade pois, como foi evidenciado, mais da metade da população mundial vive em cidades. Dentre os objetivos da agenda destaca-se os que trazem a gestão sustentável da água e saneamento, acesso sustentável à energia, crescimento econômico sustentável e tornar as cidades inclusivas (ONU, 2015).

O autor Dantas (2019) aponta o CIM como essencial para a implementação de conceitos de sustentabilidade nas cidades, colaborando assim para o cumprimento das metas estabelecidas em diversos acordos internacionais no que diz respeito à redução da atividade humana no meio ambiente, assim como a Agenda do Desenvolvimento Sustentável de 2030, citado anteriormente.

Segundo Amorim (2016a) o CIM pode servir como uma possibilidade para a solução de diversas questões decorrentes do impacto da urbanização, podendo ser viabilizado para soluções como a otimização do transporte, sistemas de água e energia, dentre outras.

Corrêa e Santos (2015) aponta que o CIM deve conter informações adequadamente representadas e georreferenciadas em um modelo 3D, servindo de apoio para o planejamento, manutenção e recuperação dos espaços urbanos. Outras ferramentas computacionais vinculadas ao CIM estabeleceriam uma conversão para uma Cidade Inteligente, sendo uma nova maneira de se gerenciar, construir, planejar e pensar em uma cidade. Amorim (2015) destaca ainda que o CIM seria uma das alternativas de se tornar uma Cidade Inteligente, que utiliza Tecnologias de Informação e Comunicação (TIC) para alcançar com efetividade uma qualidade dos sistemas urbanos e de vida dos cidadãos.

Destaca-se ainda que o CIM deve pertencer a um controle público para que realmente seja efetivo, e agregue iniciativas privadas para a sua plena execução, mas mantendo o propósito de ter uma utilidade efetiva ao município e aos cidadãos (ALMEIDA; ANDRADE, 2015). 


\section{Revista Nacional de}

Gerenciamento de Cidades

\section{REVISÃO BIBLIOGRAFICA}

Alguns autores (Almeida \& Andrade, 2018, 2016; Gil, Almeida \& Duarte, 2011) apresentaram Khemlani (2005) como sendo o precursor do CIM que tratava como uma evolução do conceito de Building Information Modelling (BIM) aplicado a cidades, para fornecer simulações por meio de uma reprodução digital da cidade, que apoiasse de maneira eficaz as decisões e análises em casos de ocorrência de desastres naturais.

O autor Amorim (2015) apresenta o CIM como uma plataforma tecnológica utilizada e apoiada por recursos humanos e conceitos de várias áreas de conhecimento, de forma integrada, para suportar a infraestrutura da cidade, atuando como suporte, operado por planejadores, projetistas e administradores, como base para a sustentação da gestão urbana.

Deste modo, as TICS se tornariam meios para aumentar a eficiência dos sistemas de energia, a prestação de serviços que variam de serviços públicos a privados nas cidades e para melhorar o transporte e a comunicação entre o poder público e a população (BATTY ET AL., 2012).

A tendência é que as cidades se transformem em plataforma de serviços, ofertando desde redes sem fio, até o atendimento de demandas da população e empresas, através de aplicações tecnológicas alinhadas a uma gestão transparente e consistente, deixando de ser apenas prestadores de serviços (WEISS; BERNARDES; CONSONI, 2017).

Almeida e Andrade (2016) revelam que o CIM possibilita que abordagens em emergências sejam tratadas de forma ordenada, viabilizando maior força para um melhor tratamento. Sendo necessário que diversas pesquisas e plataformas estejam reunidas em uma base, de forma que possa haver uma confluência de dados.

Xu et al. (2014) aponta que o foco do CIM é conquistar o compartilhamento de informações e colaboração multi-serviço e multi-campo, para alcançar o status de cidade digital e poder gerenciar completamente o espaço urbano. Tendo como as duas principais propriedades do sistema CIM, a sua interoperabilidade e o trabalho colaborativo. Devendo esse garantir a eficiência e eficácia dos serviços, mantendo uma base de dados centralizada e compartilhada, tendo que ser acessível, confiável e atualizada (AMORIM, 2016a).

Alguns autores (Amorim 2016a; Bentley 2011; Correa e Santos 2015; Gil, Almeida e Duarte 2011; Thompson et al. 2016) trataram o CIM de forma mais aplicada, demonstrando que a plataforma poderia ser desenvolvida unindo ferramentas já existentes no mercado. Por exemplo, fazendo a união de desenhos de engenharia feitos no computador (CAD), modelagem da informação da construção (BIM) e sistemas de informações geográficas (GIS). Outros autores como El-Mekawy (2010) e Van Berlo, Dijkmans, and Stoter (2013) também têm trazido avanços na pesquisa no sentido de se importar dados de um modelo Industry Foundation Classes (IFC) para um modelo City Geography Markup Language (CityGML) resultando em representações 3D da cidade, com diferentes níveis de detalhes entre as edificações. 


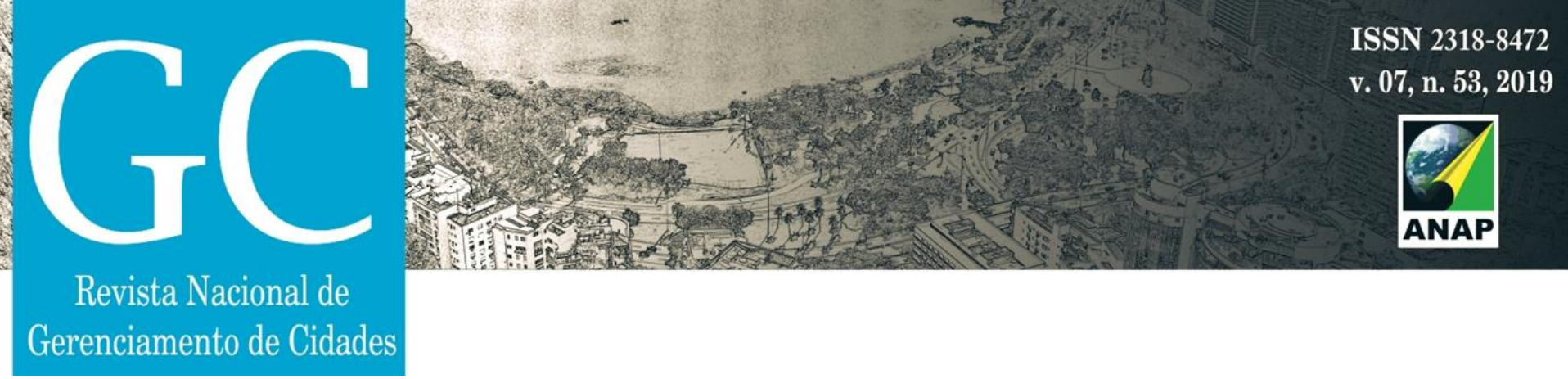

\section{OBJETIVOS}

Este trabalho tem como objetivo realizar uma análise bibliométrica da produção científica em um intervalo de dez anos (de 2009 a 2019) de pesquisas que abordem o tema CIM.

Salienta-se que o conhecimento adquirido por meio das análises bibliométricas é de grande relevância aos pesquisadores do tema, pois permitirá identificar lacunas e tendências de pesquisas guiando futuras publicações, divulgação científica e colaborações em pesquisa.

\section{METODOLOGIA}

A presente análise que tem como objetivo segundo Lopes Piñero (1972) analisar a dimensão, a evolução, a disposição da bibliografia e averiguar a estrutura dos grupos que produzem e utilizam a literatura do tema.

A pesquisa realizada neste trabalho será bibliográfica e exploratória, de acordo com Gil (2010), com intuito levantar um conhecimento disponível com teorias sobre o CIM e realizando uma revisão literária sobre o tema, tendo como objetivo "proporcionar maior familiaridade com o problema, com vistas a torná-lo mais explícito ou a construir hipóteses" (GIL, 2010). E delineada como quantitativa e descritiva, tendo em vista as definições de Miguel et al. (2012), pois terá como intuito de verificar o aspecto numérico ao analisar o número de publicações por ano, realizar o mapeamento das palavras-chave e averiguar a colaboração entre países.

Os indicadores bibliométricos foram desenvolvidos considerando os dados bibliográficos das publicações indexadas no banco de dados on-line do Scopus ${ }^{\circledR}$ é mantida pela Elsevier com acesso via https://www.Scopus ${ }^{\circledR}$. com/home.uri e Web of Science ${ }^{\mathrm{TM}}$ (WoS) é mantida pela empresa Clarivate Analytics e pode ser acessada em https://webofknowledge.com/.

As expressões de pesquisa consistiram em aplicar City Information Modelling, utilizando os operadores booleanos devido às variações dos sufixos dos termos utilizados. Que se caracterizam como operadores lógicos de uma pesquisa e sua utilização pode limitar ou ampliar os resultados obtidos.

Foi utilizado parênteses para de estipular uma relação hierárquica entre os termos de busca (CLARIVATE ANALYTICS, 2019). Além disso, foi utilizado os caracteres especiais asterisco, encarregado de manter o radical do termo de busca e retornar resultados que contenham alguma variação e as aspas que recupera os registros que contenham as palavras juntas (CAPES, 2015). Sendo assim, o termo utilizado para a busca foi ("Cit* Information Model*") em 18 de junho de 2019.

A busca na WoS e Scopus resultou em 20 e 23 registros respectivamente, devido à pequena quantidade não se utilizou refinamento, todos foram inseridos para processamento no VOSviewer. Utilizando a ferramenta de pesquisa para ocorrência em título, resumo, as palavras-chave do autor e o KeyWords Plus - que são palavras-chave relevantes destacadas pelos editores das revistas - afim de ampliar os resultados de pesquisas. 


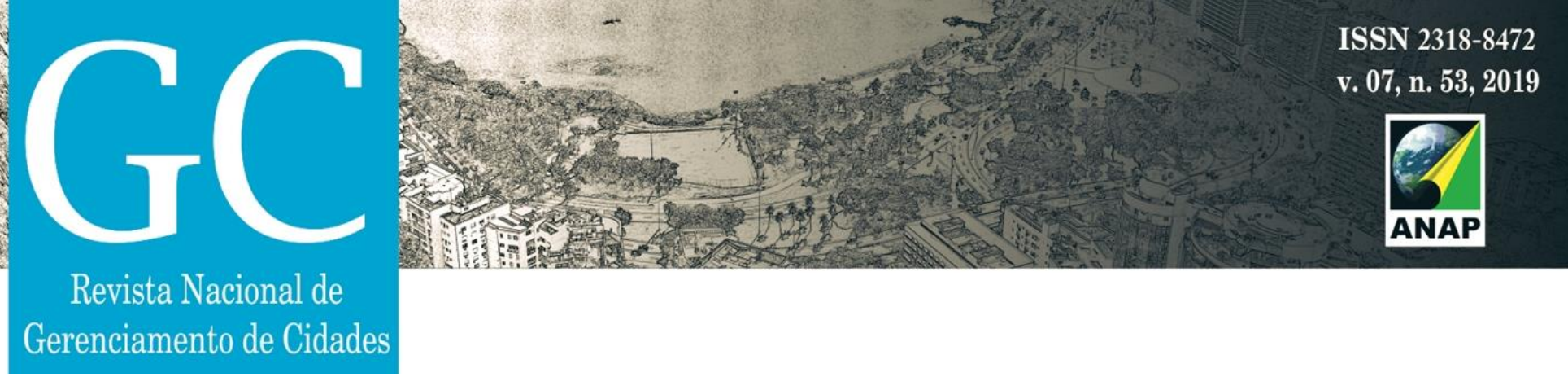

<https://www.periodicos.capes.gov.br/images/documents/Portal_Peri\%C3\%B3dicos_CAPES_Guia_2015-05-25.ppt>. Acesso em: 22 de setembro de 2019.

CLARIVATE ANALYTICS. Web of Science Core Collection Help. 2018. Disponível em: <http://images.webofknowledge.com/images/help/WOS/hs_search_operators.html\#dsy861-TRS_booleans>. Acesso em: 15/08/2019.

CORRÊA, F. R.; SANTOS, E. T. Na direção de uma modelagem da informação da cidade (CIM). In: Anais VII Encontro de Tecnologia de Informação e Comunicação na Construção (TIC 2015). Porto Alegre: ANTAC, 2015.

DANTAS, H. S.; SOUSA, J. M. M. S.; MELO, H. C. The Importance of City Information Modeling (CIM) for Cities' Sustainability. In: IOP Conference Series: Earth and Environmental Science. IOP Publishing, 2019. p. 012074.

EL-MEKAWY, M. Integrating BIM and GIS for 3D city modelling: The case of IFC and CityGML. Stockholm, Sweden: KTH, 2010. 70 p.

EL-MEKAWY, M. Integrating BIM and GIS for 3D city modelling. The Case of IFC and CityGML. Sweden: Royal Institute of Technology, 2010.

GIL, A. Como Elaborar projetos de pesquisa, 5a Edição, São Paulo: Editora Atlas, 2010.

GIL, J.; ALMEIDA, J.; DUARTE, J. The backbone of a City Information Model (CIM): Implementing a spatial data model for urban design. In: Proceedings EDUCATION AND RESEARCH IN COMPUTER AIDED ARCHITECTURAL DESIGN IN EUROPE, 29., 2011, Ljubljana. University of Ljubljana / Faculty of Architecture, 2011.

KHEMLANI, L. City Information Modeling. AECbytes, 2016. Disponível em: <http://www.aecbytes.com/feature/2016/CitylnformationModeling.html>. Acesso em: 11 de abril de 2019

LÓPEZ PIÑERO, J. M. El análisis estadístico y sociométrico de la literatura científica. Valencia: Centro de documentación e informática médica, v. 197, p. 2007-2012, 1972.

MIGUEL, P. A. C. Metodologia de Pesquisa em Engenharia de Produção e Gestão de Operações. 2.a ed. Rio de Janeiro: Elsevier, 2012.

ONU - ORGANIZAÇÃO DAS NAÇÕES UNIDAS. World Urbanization Prospects, The 2011 revision. New York, 2012. Disponível

<https://www.un.org/en/development/desa/population/publications/pdf/urbanization/WUP2011_Report.pdf>. Acesso em 20 de abril de 2019.

ONU - ORGANIZAÇÃO DAS NAÇÕES UNIDAS. Transformando Nosso Mundo: A Agenda 2030 para o Desenvolvimento Sustentável. New York, 2015. Disponível em:<https://nacoesunidas.org/pos2015/agenda2030/>. Acesso em: 15 de abril de 2019.

THOMPSON, E. M.; SMITH, K. M.; CHARLTON, J.; DOLNÍK, M. Planners in the future city: using city information modelling to support planners as market actors. Urban Planning, v. 1, n. 1, p. 79-94, 2016.

VAN BERLO, L. A. H. M.; DIJKMANS, T.; STOTER, J. E. Experiment for inegrating Dutch 3D spatial planning and BIM for checking building permits. In: 8th 3DGeolnfo Conference \& WG II/2 Workshop, Istanbul, Turkey, 27-29 November 2013, ISPRS Archives Volume II-2/W1. ISPRS, 2013.

WEISS, M. C.; BERNARDES, R. C.; CONSONI, F. L. CIDADES INTELIGENTES: casos e perspectivas para as cidades brasileiras. Revista Tecnológica da Fatec Americana, v. 5, n. 1, p. 01-13, 2017.

XU, X.; DING, L.; LUO, H.; MA, L. From Building Information Modeling to City Information Modeling. Journal of Information Technology in Construction, v. 19, p. 292-307, 2014. 